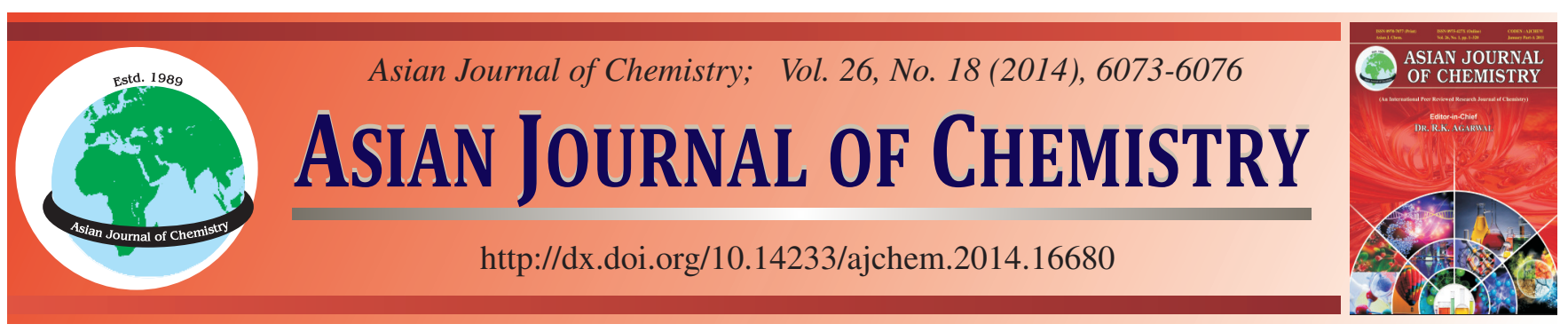

\title{
Separation of Three Flavones from Chamaecyparis obtusa Using Functionalized Hollow Microsphere Polymers
}

\author{
Minglei Tian, Wentao Bi and Kyung Ho Row*
}

Department of Chemistry and Chemical Engineering, Inha University, 253 Yonghyun-Dong, Nam-Ku, Incheon 402-751, Republic of Korea

*Corresponding author: E-mail: rowkho@inha.ac.kr

Received: 12 November 2013;

Accepted: 14 March 2014;

Published online: 1 September 2014;

AJC-15856

Soxhlet extractor was used to extract three flavones (quercitrin and myricetin and amentoflavone) from Chamaecyparis obtusa. A cartridge packed with sorbent was fixed at the end of the siphon side arm. The sorbent was used to absorb the target compounds during the extraction process. In order to obtain the highest separation efficiency, several functionalized ionic liquid-immobilized hollow microsphere polymers were investigated. Finally, the amino ionic liquid-immobilized hollow microsphere polymer with highly selectivity and stability achieved the purpose and $0.44 \mathrm{mg} \mathrm{g}^{-1}$ of quercitrin, $0.19 \mathrm{mg} \mathrm{g}^{-1}$ of myricetin and $0.12 \mathrm{mg} \mathrm{g}^{-1}$ of amentoflavone were obtained.

Keywords: Flavone, Chamaecyparis obtusa, Hollow microsphere polymer, Soxhlet extraction.

\section{INTRODUCTION}

Chamaecyparis obtusa is one of the genus Chamaecyparis and is distributed mainly in Asian countries. Flavones are the other main compounds in Chamaecyparis obtusa, including quercitrin, myricetin and amentoflavone (Fig. 1), which have antioxidant and anticancer abilities. Quercetrin is used as an antibacterial agent and has been found to inhibit the oxidation of low-density lipoproteins ${ }^{1,2}$. Previous studies ewported that myricetin has an antiproliferative effect on lung cancer cells ${ }^{3}$. The antiangiogenic activity of amentoflavone might help further improve its effectiveness in controlling cancer growth and metastasis ${ }^{4}$. In order to increase the therapeutic effect of the flavones as drug, they should be extracted, concentrated and purified. Soxhlet extraction is one of the most efficient methods to extract flavones from plants ${ }^{5,6}$. One the other hand, interference compounds are also extracted with the target compounds.

This study examined an improvement of extraction. During extraction, the chamber was emptied automatically through a siphon side arm. A cartridge packed with sorbent was fixed at the end of the siphon side arm. As the extraction solvent repeatedly runs back down to the distillation flask many times, the sorbent can absorb the target compounds and separate them from interference.

Hence, the properties of the sorbent are crucial to the performance of this method. During previous researches, polymers

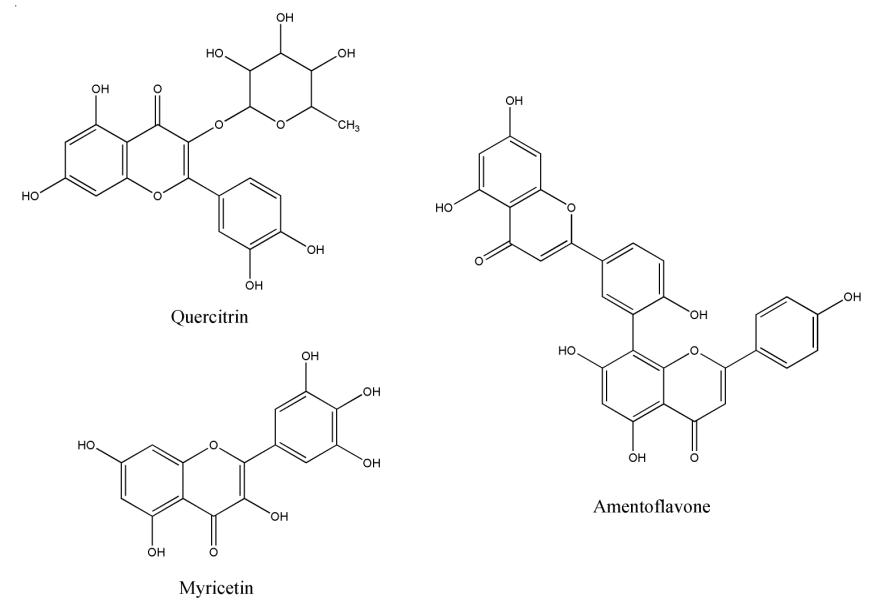

Fig. 1. Chemical structure of three flavones from Chamaecyparis obtusa

had been reported as the separation sorbent, but the efficiency was not high enough ${ }^{7}$. Among all polymer materials, the hollow microsphere polymer (HMP) has attracted increasing attention because of its excellent mechanical properties and good chemical stability over a wide $\mathrm{pH}$ range ${ }^{8}$. Hollow microsphere polymers have already been applied in separation because of their specific structure, pore size and functional groups from the monomer. On the other hand, the adsorption, selectivity and affinity of flavones on unmodified microsphere polymer are very difficult to control. Functional groups modification 
of the polymer surface is essential to increase the separation efficiency ${ }^{9}$. Ionic liquids, a green reaction media with excellent chemical properties, have been employed as a modifier. Ionic liquids have attracted considerable attention in analytical chemistry, sample preparation, organic synthesis, liquid-phase extraction and chromatographic separations. The characteristics, such as hydrophobicity, miscibility with several inorganic/organic solvents, $\pi-\pi$ interactions between the analyte and functional groups of the ionic liquids, are applied widely ${ }^{10}$. In this case, hollow microsphere polymer will be modified by ionic liquids to enhance its selectivity and applied in Soxhlet extraction.

\section{EXPERIMENTAL}

Quercitrin ( $\geq 78 \%)$, myricetin $(\geq 96 \%)$ and amentoflavone $(\geq 99 \%)$ were obtained from Sigma-Aldrich (St. Louis, MO, USA). Organic solvents were obtained from Duksan Pure Chemical Co., LTD (Ansan, Korea). All the other reagents used in the experiment were HPLC or extra pure grade. The silica particles $(\geq 7 \mu \mathrm{m})$ were purchased from SUNJIN Chemical Co. Ltd. and a specific surface is $300 \mathrm{~m}^{2} / \mathrm{g}$. For synthesis of polymer, 3-chloropropyltriethoxysilane (>97\%), benzoyl peroxide $(>75 \%)$, poly(vinyl alcohol) $(\mathrm{n} \approx 2000)$, divinylbenzene $(\geq 50 \%)$, 4-(chloromethyl)styrene $(>90 \%)$ and several kinds of imidazole with different functional groups $(\geq$ $95 \%$ ) were supplied by TCI (Tokyo, Japan). Double distilled water was filtered with a Waters vacuum pump (Billerica, USA). All samples were filtered (Advantec, $0.2 \mu \mathrm{m}$, Tokyo, Japan) before being injected into the HPLC system.

HPLC analysis: HPLC was performed using a Waters 600s multisolvent delivery system, a Waters 616 liquid chromatography and a Waters 2487 variable wavelength, dualchannel UV detector (Milford, USA). A commercial $\mathrm{C}_{18}$ column $(4.6 \times 250 \mathrm{~mm}, 5 \mu \mathrm{m})$ purchased from RStech Co. (Daejeon, Korea) was used. The mobile phase, flow rate, UV wavelength and injection volume was acetonitrile/water $(35: 65,+0.1 \%$ TFA, v/v), $0.5 \mathrm{~mL} \mathrm{~min}^{-1}, 372 \mathrm{~nm}$ and $5 \mu \mathrm{L}$, respectively. The three target compounds were dissolved in methanol $(0.1 \mathrm{mg}$ $\mathrm{g}^{-1}$ ) for further analysis.

The reproducibility was assayed by the relative standard deviation (RSD) which was determined by injecting standard solutions 5 times over a 5-day period. The RSDs of the precision tests were $=3.32 \%$ of the standard.

Preparation of hollow polymers: $10 \mathrm{~g}$ silica was stirred with 3-chloropropyltrimethoxysilane $(5 \mathrm{~mL})$ and then refluxed in $50 \mathrm{~mL}$ dry toluene. After $24 \mathrm{~h}$ reaction, the powders were washed with ethanol.

Silanized silica (3 g) and aqueous poly(vinyl alcohol) $(30 \mathrm{~mL})$ were added to a flask and the mixture was aerated with $\mathrm{N}_{2}$ gas for $0.5 \mathrm{~min} .3 \mathrm{~mL}$ of the mixed monomer solutions consisting of 4-(chloromethyl)styrene $(0.7 \mathrm{~g})$, divinylbenzene $(2 \mathrm{~g})$ and benzoyl peroxide $(0.15 \mathrm{~g})$ was added to the flask. The mixture was stirred for $24 \mathrm{~h}$ at room temperature to allow the monomer and crosslink enter into the pore of silica. Then mixture was heated at $90^{\circ} \mathrm{C}$ and the polymerization was finished after $12 \mathrm{~h}$. After that, the power was washed with water and methanol, it was stirred in a mixture of a $5 \mathrm{~mol} / \mathrm{L}$ of aqueous solution of $\mathrm{NaOH}$ and methanol for $24 \mathrm{~h}$ at room temperature to dissolve the silica. At last, it was washed with water until the solution was neutral followed by $100 \mathrm{~mL}$ of methanol.

After the substrate was obtained, $0.3 \mathrm{~g}$ of the polymer was modified with different kind of imidazoles $(0.014 \mathrm{~mol})$ in ethanol for $12 \mathrm{~h}$ under $75^{\circ} \mathrm{C}$. At last, the obtained polymers (Fig. 2) were washed by ethanol for several times and overdired for further experiment.
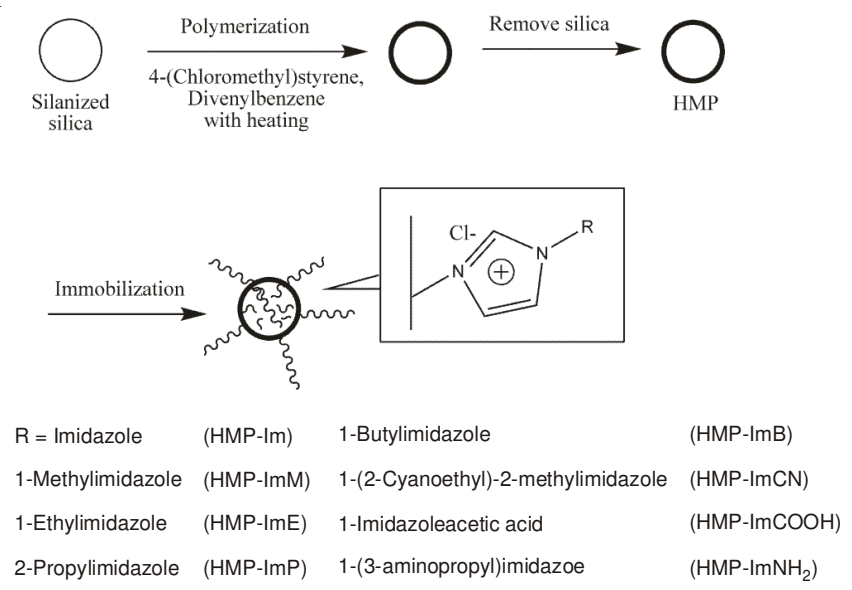

Fig. 2. Preparation scheme of all functionalized hollow microsphere polymers

Adsorption isotherm application: $0.01 \mathrm{~g}$ of the polymers and $1 \mathrm{~mL}$ of standard solutions of the three flavones at 5 different concentrations $\left(0.05-0.2 \mathrm{mg} \mathrm{mL}^{-1}\right)$ were added to the vials with constant stirring under room temperature. When equilibrium adsorption was obtained, the amounts of flavones adsorbed on the polymers were calculated by subtracting the concentrations of unadsorbed compounds remaining in solutions.

\section{RESULTS AND DISCUSSION}

Calibration curves were constructed using the chromatographic peak areas measured at seven concentrations, ranging from $1 \times 10^{-5}$ to $0.3 \mathrm{mg} \mathrm{mL}^{-1}$. Good linearity was obtained with the following linear correlation equations: $Y=214160 \mathrm{X}-$ $2834.6\left(r^{2}=0.999\right)$ for quercitrin, $\mathrm{Y}=448849 \mathrm{X}-46692\left(\mathrm{r}^{2}=\right.$ $0.999)$ for myricetin and $Y=246289 X-22376\left(r^{2}=0.999\right)$ for amentoflavone ( $\mathrm{Y}$ is the peak area and $\mathrm{X}$ is the concentration of target compounds).

Amounts $(\mathrm{Q})$ of flavones adsorbed on all the sorbents were determined using the following eqn:

$$
\mathrm{Q}=\frac{\left(\mathrm{C}_{0}-\mathrm{C}\right) \mathrm{V}}{\mathrm{m}}
$$

where $\mathrm{Q}\left(\mathrm{mg} \mathrm{g}^{-1}\right)$ is the amount adsorbed, $\mathrm{C}_{0}\left(\mathrm{mg} \mathrm{mL}^{-1}\right)$ is the initiator concentration, $\mathrm{C}\left(\mathrm{mg} \mathrm{mL}^{-1}\right)$ is the unabsorbed concentration, $\mathrm{V}(\mathrm{mL})$ is the volume of the sample solvent and $\mathrm{m}(\mathrm{g})$ is the mass of the sorbent. Fig. 3 ( $a, b$ and c) shows all the amounts adsorbed.

The hydrophilic/hydrophobic characteristic of the sorbent and the interactions among functional groups have major effects on the adsorption ability. All the target compounds exhibited hydrophilic characteristics because of the large number of -OH groups. When commercial sorbents were used, $\mathrm{C}_{18}$ had the highest hydrophobicity which means it was virtually unable to adsorb the target compounds. Silica (hydrogen-bonding 

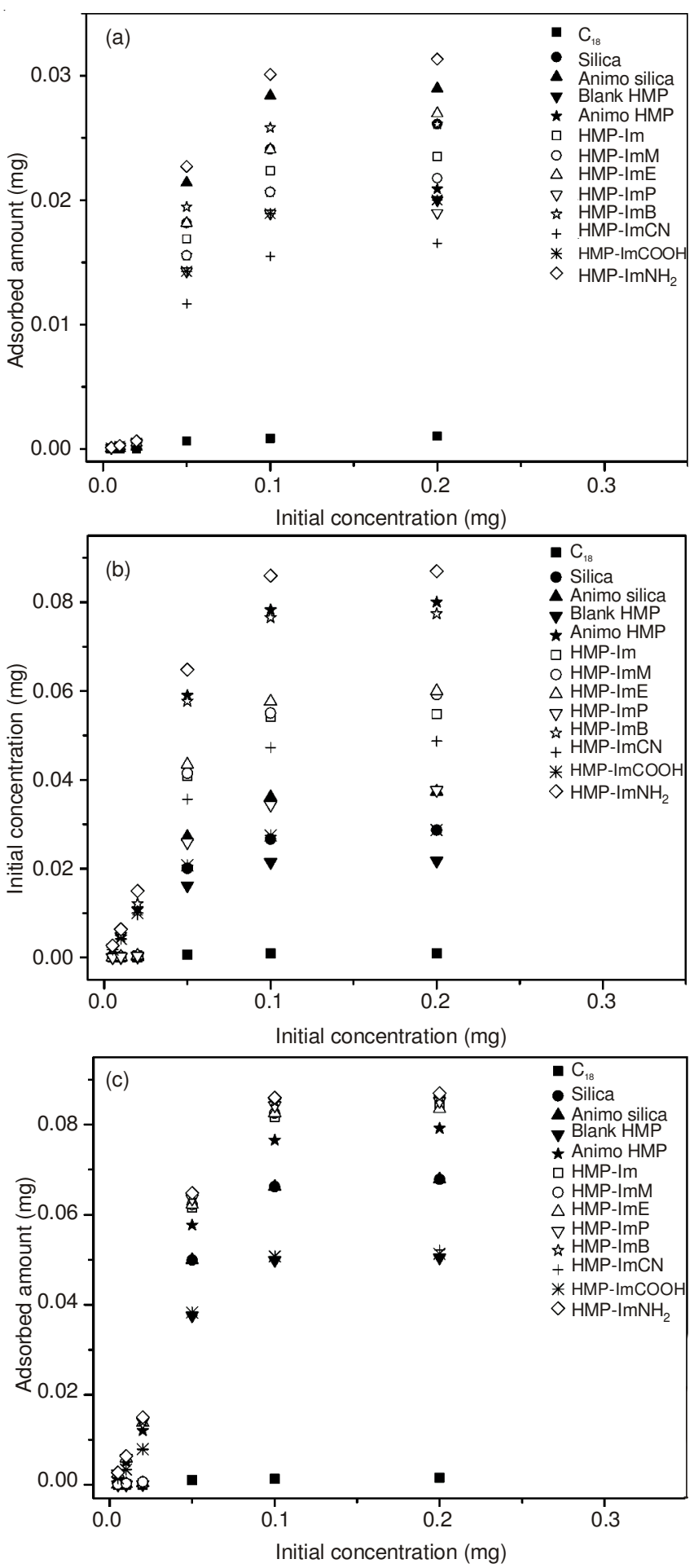

Fig. 3. Adsorbed amount of (a) quercitrin, (b) myricetin and (c) amentoflavone on different sorbents

forms) could absorb only limited amounts of the target compounds owing to its hydrophilicity. On the other hand, larger amounts of the target compounds were absorbed when amino groups were added (amino-silica). This shows that the additional functional groups resulted in more interactions with the target compounds.

For the polymer sorbents, even the blank polymer could absorb some of the targets compounds owing to its porous structure. The amounts absorbed increased significantly, when the polymers contained immobilized ionic liquid group due to the $\pi-\pi$ and hydrophilic interactions (HMP-Im, HMP-ImM, HMP-ImE, HMP-ImP and HMP-ImB). The densities of the imidazolium-based ionic liquids decreased with increasing carbon chain length. Therefore, ionic liquids with longer alkyl chains were expected to have greater free volume and interact with larger amounts of the target compounds. However, the hydrophobicity of the ionic liquid increased with increasing carbon chain length. In this case, the adsorption ability showed a limited increase with increasing carbon chain length on the ionic liquid polymer.

The interaction of different functional groups on the ionic liquid group was examined by comparing the effects of HMPImCN, HMP-ImCOOH and HMP-ImNH ${ }_{2}$. The ionic liquid with amino groups showed the highest interaction with all the target compounds examined. A polymer with amino groups (amino HMP) was only used for confirmation and showed lower adsorption ability. Therefore, $\mathrm{HMP}-\mathrm{ImNH}_{2}$ was selected as the sorbent.

Comparison between different types of sorbents: Table-1 showed the absorbed amount of target compounds on different types of sorbents. On the adsorption ability, the structure of polymer had highly effect. Hollow polymer contained large amount of functional groups with large size of pore, so as many as functional groups can interact with target compounds. In this case, HMP-ImNH$H_{2}$ had the largest adsorption amount.

\begin{tabular}{cccc} 
& \multicolumn{3}{c}{ TABLE-1 } \\
COMPARISON OF ADSORBED AMOUNT OF THREE \\
FLAVONES ON DIFFERENT TYPES OF SORBENTS
\end{tabular}

Washing and elution of target compounds from cartridge: The cartridge (packed with $0.2 \mathrm{~g}$ of sorbents) was removed and different solvents were used to separate target compounds from interference. Washing can reduce the interference during separation of the analyte. During the Soxhlet extraction, different solvents (dichloromethane, $n$-hexane, water, methanol, ethanol and acetonitrile) were used to obtain the highest amount of target compounds from C. obtusa. Methanol can extract the highest amount $\left(0.44 \mathrm{mg} \mathrm{g}^{-1}\right.$ of quercitrin, $0.19 \mathrm{mg} \mathrm{g}^{-1}$ of myricetin and $0.12 \mathrm{mg} \mathrm{g}^{-1}$ of amentoflavone) and $n$-hexane 
obtained no target compounds. In this case, $n$-hexane was selected as the washing solvent and $4 \mathrm{~mL}$ showed no further removal of any interference. Therefore, $4 \mathrm{~mL}$ of $n$-hexane was considered optimal for washing.

Water was firstly used to elute quercitrin from HMP$\mathrm{ImNH}_{2}$ because of the hydrophilicity of quercitrin and the strong interaction between myricetin, amentoflavone and sorbent. The level of quercitrin eluted by water increased with increasing volume to $2 \mathrm{~mL}$ with no further elution at higher volumes. Hence, $2 \mathrm{~mL}$ of water was considered to be the first elution solvent. Methanol was selected in the second elution stage due to the characteristics of myricetin and amentoflavone. When $4 \mathrm{~mL}$ of methanol was used, all the myricetin was eluted out. Therefore, $4 \mathrm{~mL}$ of methanol was selected as the second elution solvent. The final stage was to elute the amentoflavone from the HMP-ImNH $\mathrm{H}_{2} .2 \mathrm{~mL}$ of methanol/1\% acetic acid (vol. $\%$ ) with strong elution ability was used to break the interaction between the target compounds and sorbent. In conclusion, low volume of washing $(4 \mathrm{~mL})$ and elution solvent (each time less than $4 \mathrm{~mL}$ ), high selectivity of interactions demonstrated the target compounds were successfully separated (Fig. 4).

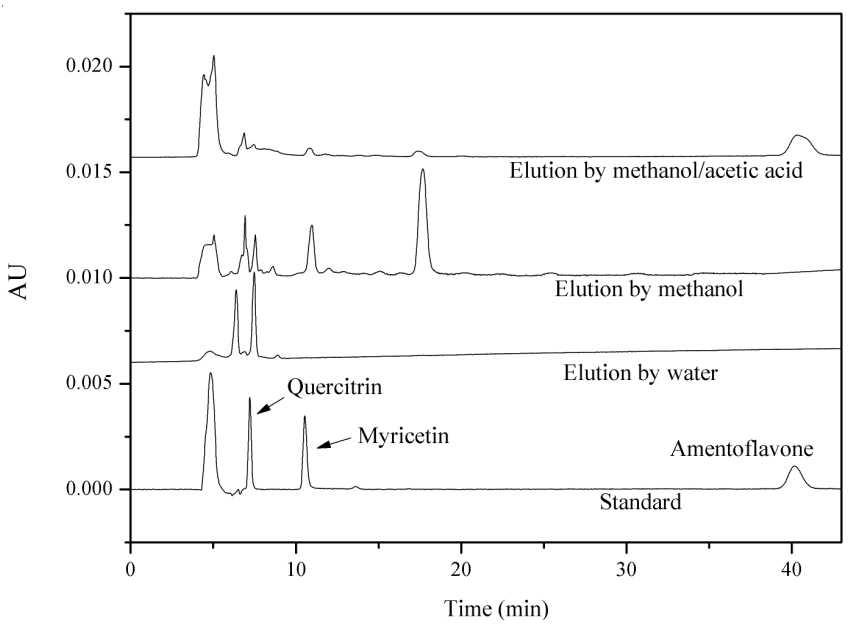

Fig. 4. Chromatograms of three flavones from standards, extraction, washing and elution stages
Recycling of sorbent: Validation of the method and recycling of the sorbent cartridge were investigated simultaneously. The errors in detected concentrations of target compounds were less than $3 \%$ demonstrating the stability of the sorbent. Finally, under optimized conditions, $0.44 \mathrm{mg} \mathrm{g}^{-1}$ of quercitrin, 0.19 $\mathrm{mg} \mathrm{g}^{-1}$ of myricetin and $0.12 \mathrm{mg} \mathrm{g}^{-1}$ of amentoflavone were obtained with $0.2 \mathrm{~g}$ of sorbents.

\section{ACKNOWLEDGEMENTS}

This research was supported by Basic Science Research Program through the National Research Foundation (NRF) of Korea funded by the Ministry of Education, Science and Technology (2014-002046).

\section{REFERENCES}

1. J.R. Soberon, M.A. Sgariglia, D.A. Sampietro, E.N. Quiroga and M.A.J. Vattuone, Appl. Microbiol., 102, 1450 (2007).

2. A.S. Meyer, M. Heinonen and E.N. Frankel, Food Chem., 61, 71 (1998).

3. J. Lu, L.V. Papp, J. Fang, S. Rodriguez-Nieto, B. Zhivotovsky and A. Holmgren, Cancer Res., 66, 4410 (2006).

4. C. Guruvayoorappan and G. Kuttan, Biochemistry (Moscow), 73, 209 (2008).

5. M. Gao and C. Liu, World J. Microbiol. Biotechnol., 21, 1461 (2005).

6. M. Waksmundzka-Hajnos and J. Sherma, High Performance Liquid Chromatography in Phytochemical Analysis, Taylor \& Francis Group, New York (2010).

7. M. Tian, D. Han and K.H. Row, Anal. Lett., 44, 737 (2011).

8. B. Wei, S. Wang, H. Song, H. Liu, J. Li and N. Liu, Petrol. Sci., 6, 306 (2009).

9. W. Bi, M. Tian and K.H. Row, J. Sep. Sci., 33, 1739 (2010).

10. M. Tian and K.H. Row, Chromatographia, 73, 25 (2011). 